REVISTA ECONOMÍA

Vol. 7I, N. ${ }^{\circ}$ II3 (mayo 20I9), 45-59

\title{
DISCRIMINACIÓN SALARIAL ECUATORIANA POR RAZÓN DE GÉNERO Y AUTOIDENTIFICACIÓN
}

\author{
BRYAN ESPINOZA ESTRELLA, ${ }^{,}$RAMIRO VILLARUEL MEYTHALER,${ }^{2}$ \\ YLENIA QUINTANA SALDARRIAGA ${ }^{3}$ \\ ${ }^{1,3}$ Universidad Central del Ecuador, ${ }^{2}$ Pontificia Universidad Católica del Ecuador
}

Fecha de envío: 25 de abril de 2019

Aceptación de versión final: 30 de abril de 2019

\begin{abstract}
RESUMEN Con el establecimiento de una nueva Constitución en el 2008, en Ecuador se evocan conceptos de derechos e igualdad, igualdad de género, diversidad laboral y trabajo doméstico, entre otros. Sobre ello, específicamente en el campo de la igualdad de género, la intención de este trabajo es analizar a través de dos modelos, de manera respectiva, si existe o no discriminación salarial por razón de género así como discriminación salarial femenina por autoidentificación. Luego de analizar los resultados, se encuentra que sí existe discriminación salarial entre hombres y mujeres; y dentro del campo femenino se observa discriminación salarial por razón étnica.
\end{abstract}

PALABRAS CLAVes Discriminación, igualdad, autoidentificación, género.

ABSTRACT With the establishment of a new Constitution in 2008, in Ecuador, concepts of rights and equality, gender equality, labor diversity and domestic work are established, among others. In the field of gender equality, the intention of this work is to analyze, in two models, respectively, whether or not there is wage discrimination based on gender, as well as female wage discrimination for self-identification. After analyzing the results, it is found that there is wage discrimination between men and women; and within the female field, wage discrimination based on ethnicity is observed.

KEYWORDS Discrimination, equality, self-identification, gender.

JEL CODE B23; J15; J16.

\section{INTRODUCCIÓN}

Desde el año 2000, el Estado ecuatoriano propuso nuevas concepciones y reglas alrededor de temas como trabajo, economía, derechos e igualdad de oportunidades para todos los ciudadanos. A la vez, en el año 2008, la Asamblea Constituyente planteó una nueva Constitución con un enfoque más integrador, donde se incluyeron temas como igualdad de género, reconocimiento de la diversidad laboral y también el trabajo doméstico con su respectiva remuneración, entre otros (Rodríguez, 2016). Sobre este amplio marco, en el tema de igualdad de 
género, la pregunta que surge es si existe o no en el país diferencia salarial entre hombres y mujeres; y dentro del campo femenino, si existe o no diferencias salariales por razón étnica. En el caso de que existan se torna importante estudiar y medir estas diferencias; y con ello, presentar un documento técnico por el cual se puedan direccionar de mejor manera las políticas públicas en este campo.

Un estudio realizado por onU Mujeres, en el año 2016 concluyó que la discriminación salarial es generalizada en América Latina y el Caribe, y está vinculada con otros aspectos adicionales como los socioeconómicos y autoidentificación étnica (onU Mujeres, 2017). En tal sentido, tanto la condición de mujeres como de la población que no sea parte del grupo etario predominante, mestizas en el caso de Ecuador, pueden estar relacionadas con esta discriminación salarial y laboral. Una de las razones por las que se quiere entender los fenómenos de discriminación salarial es que explicarían en gran medida procesos de pobreza y exclusión social. En este sentido, cabe resaltar que la calidad de vida de una familia está relacionada con el ingreso salarial, y estas pérdidas económicas conllevan a mermar el desarrollo social y humano.

\section{MARCO TEÓRICO}

Las visiones teóricas que tratan acerca de la discriminación por género parten como principal problema de que las mujeres han sido relegadas hacia la organización familiar y al trabajo doméstico. Desde estas visiones, se ha intentado comprender sus características y, con ello, sacar inferencias y conclusiones que intenten desterrar todo tipo de discriminación. En la actualidad, se han desarrollado una serie de conceptos que vale la pena plantearlos desde un principio para una mejor clarificación. Con respecto a la discriminación salarial se da cuando dos personas perciben salarios diferentes con la misma productividad. Este tipo de discriminación puede encauzarse a través de varias formas, entre ellas se encontrarían por género, edad, procedencias, religión, nacionalidad, entre otras.

Otro de los conceptos que vale manejar es la de discriminación en el empleo, que se da cuando una persona es tratada de forma diferente a causa de su autoidentificación, sea por género o religión, es decir, aquí no se aplican conceptos salariales, sino en su trato o en el tipo de trabajo que se le asigna. Acerca de la brecha salarial, de acuerdo a Eurostat Static Explained (2017), es la diferencia porcentual que existe entre el salario medio de hombres y el salario medio de mujeres en relación al de hombres cuando se realiza la misma actividad. Entre los factores que explicarían este fenómeno, estarían la segregación sectorial y profesional, educación y formación, sensibilización y transparencia, además de la discriminación directa. Entre las causas que explicarían la brecha salarial o la discriminación salarial por razón de género, es la situación para la mujer de conciliar su trabajo con su vida privada.

Para hacer llevadera esta realidad, las mujeres buscan trabajos a tiempo parcial, lo que las empuja a la vez a contratos atípicos; si bien esto les permite estar integradas en el mercado laboral, también les genera un impacto negativo en la evolución de sus carreras, ascensos y pensiones. Bajo esta situación descrita, cualquier grupo es más propenso a caer o mantenerse dentro de estos círculos viciosos de pobreza, por la misma condición de percibir salarios menores. Otras razones se atañan al mismo empresario. Para Becker (1971), existe el gusto por la 
Figura 1. Tasa de empleo adecuado por género

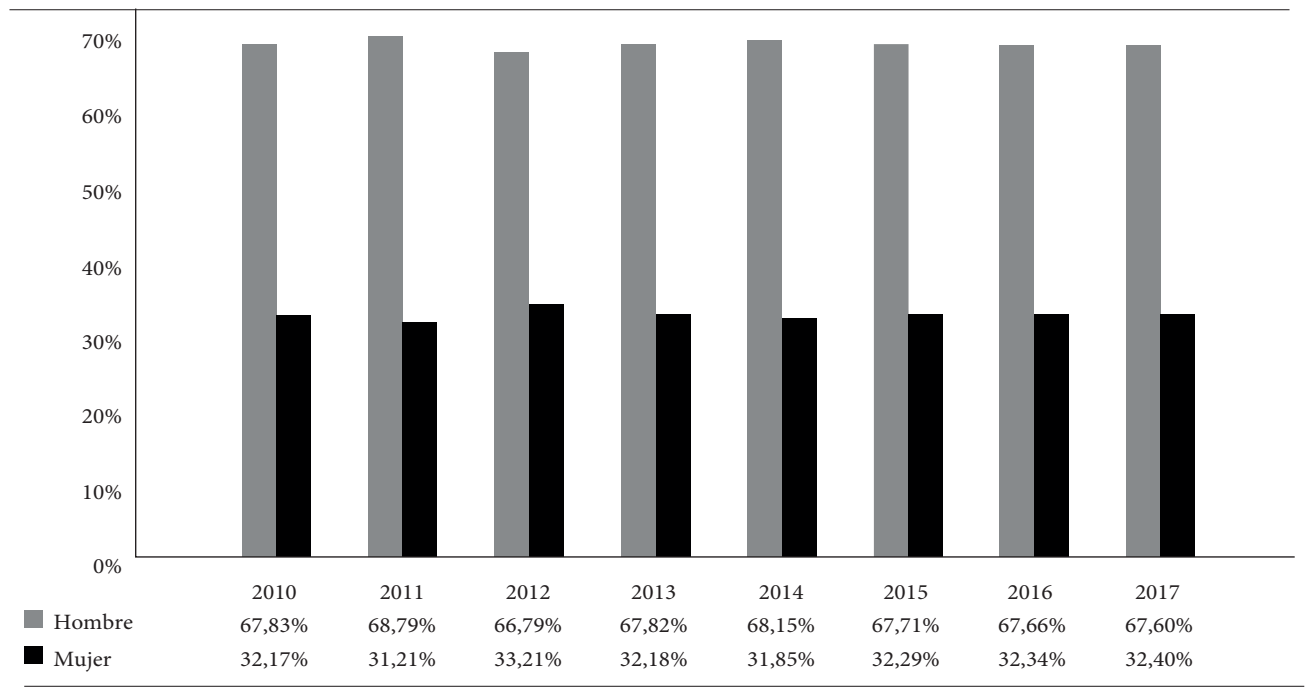

Fuente: ENEMDU (2010-2017), INEC (en línea). Elaboración: propia.

Figura 2. Tasa de subempleo por género

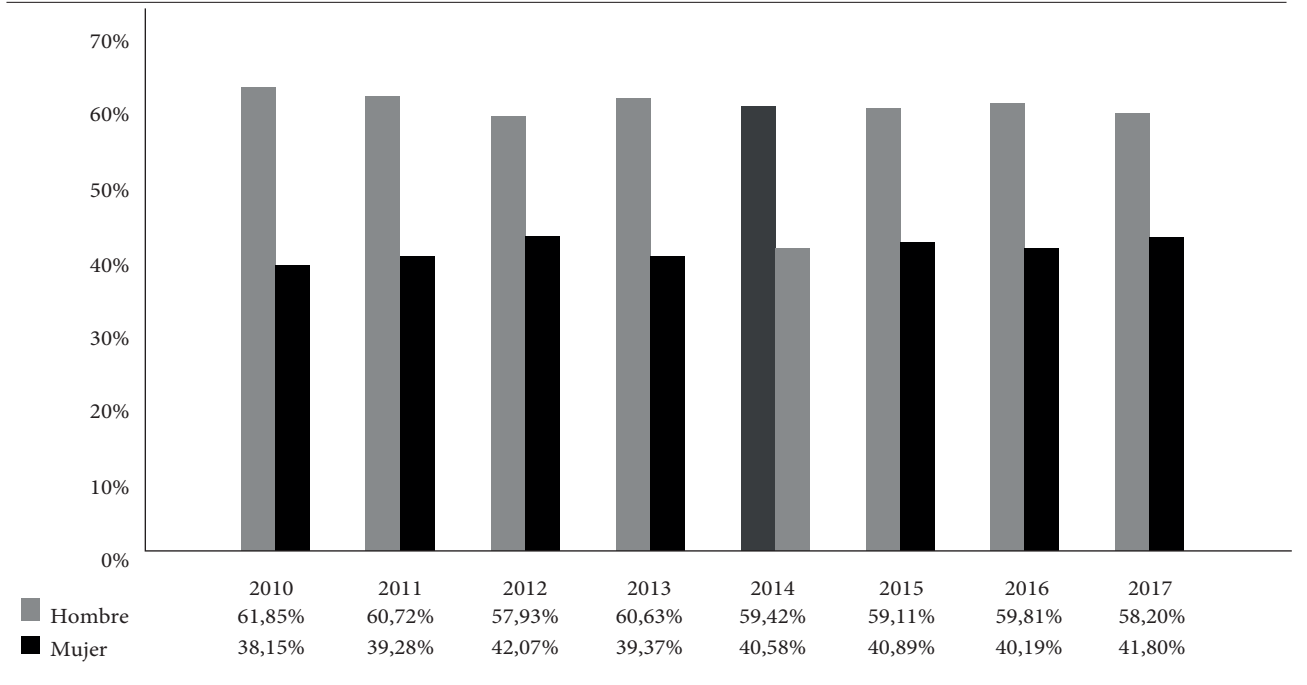

Fuente: ENEMDU (2010-2017), INEC (en línea). Elaboración: propia.

discriminación; su punto de vista parte de que los empresarios discriminan a la hora de contratar personal, es decir, un individuo será contratado si está dispuesto a recibir un menor salario. Con respecto a las mujeres, según Becker (ibíd.), el empleador arrastra un costo psíquico o adicional, y hace referencia al hecho de la posibilidad de embarazo o ausentismo laboral por preocupaciones familiares.

Desde esta óptica, los empresarios solo emplearán a mujeres cuando acepten un salario 
menor porque, a diferencia del hombre, en ella se cifrarían todos los inconvenientes que supone el contratarla. No obstante, al final, esto ocasiona costos y pérdidas de eficiencia en la producción al no contratarse personal indicado. Dentro de este marco, el dinero sirve como una medida de discriminación y lleva en sí un costo para el empleador denominado coeficiente de discriminación. Por otro lado, existe la discriminación estadística propuesta por Aigner y Cain (1977). La visión supone que los empresarios toman como referencia, para juzgar a una persona, las características promedio del grupo al que pertenecen, ya que no poseen información detallada del individuo, pero sí del grupo. En este sentido, los autores proponen que la mujer cae en este tipo de discriminación, ya que se la vincularía con un carácter más sensible, limitada en fortaleza física y, entre otras, con el rol de amas de casa, con preocupaciones de hijos y familia en general.

Otra de las razones de la existencia de discriminación salarial por razón de género se daría por segregación ocupacional, o que también podría llamarse de segregación laboral, con el fin de encauzar varios escritos que llaman al mismo concepto. Para Gross (1968), el empresario considera que algunos grupos tendrán menor productividad que otros. Así entonces, al hablar de hombres y mujeres se tratarían de dos fuerzas laborales diferentes, la masculina y femenina, con lo cual se excluye a un género. Peñas (1998), en este sentido, añade que la segregación laboral puede ser de dos tipos: la primera, del tipo horizontal, que se produce cuando las personas encuentran dificultades para el acceso a ciertas profesiones; la segunda, del tipo vertical, que se presenta cuando se encuentran dificultades para ascender en la carrera profesional -observada especialmente en el caso de las mujeres-, puesto que no suelen ocupar cargos elevados y de mayor importancia.

Con respecto a la evolución histórica se podría añadir que siempre han existido grupos sociales que se han autodefinido como seres superiores, ya sea por su tono de piel, preferencia sexual, género o condición social. Bajo esta premisa, estos grupos han promovido situaciones tan incómodas como la discriminación y desigualdad, lo que consecuentemente ha generado que en la sociedad no sea fácil la implementación de medidas contrarias. De hecho, los primeros mecanismos de discriminación, xenofobia y racismo fueron la esclavitud y la trata de esclavos. Keegan (1994), sostiene que no es sencillo esclarecer una fecha de inicio para este tipo de prácticas atroces, ya que formaban parte del ordenamiento territorial en la antigüedad. Historiadores como Hopenhayn y Bello (2000), en relación a la época de la conquista y colonización, aseveran que tanto el dominio de territorios, apropiación de la riqueza de las comunidades afrodescendientes e indígenas, así como la imposición de creencias religiosas y políticas fueron también formas de sumisión y de discriminación de estos grupos.

Ya en el año 2001, en la Conferencia Mundial de las Naciones Unidas sobre Racismo, Discriminación, Xenofobia y otras formas de intolerancia, se reconoció que estas prácticas intransigentes son graves violaciones a los derechos humanos, y que «[...] constituyen un obstáculo a las relaciones pacíficas y de amistad entre los pueblos y las naciones, y figuran entre las causas básicas de muchos conflictos internos e internacionales [...]» (Naciones Unidas, 2001). Dentro de esta conferencia, como puntos finales, se concluyó conjuntamente que la segregación, discriminación y racismo generan un trato de inferioridad a un grupo de personas simplemente por sus diferentes características físicas, socioeconómicas y culturales. 
Figura 3. Tasa de desempleo por género

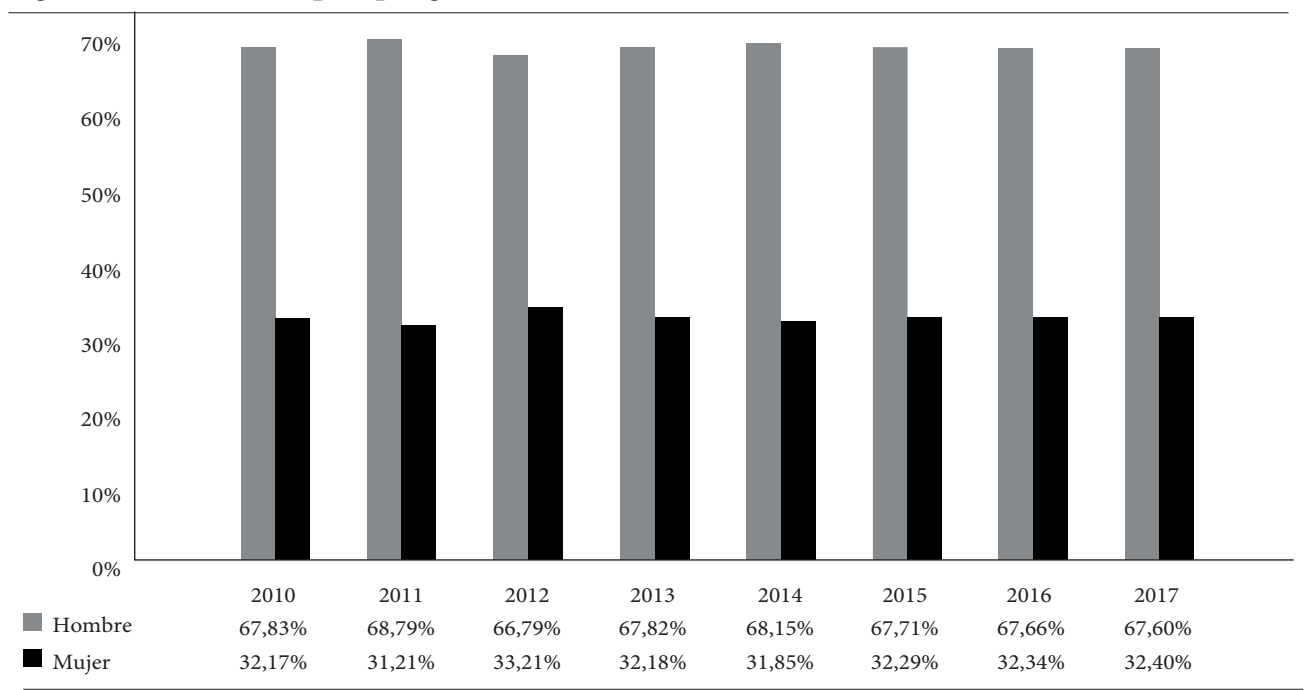

Fuente: ENEMDU (2010-2017), INEC (en línea). Elaboración: propia.

\section{CONDICIONES LABORALES POR GÉNERO EN ECUADOR}

Desde el surgimiento de la sociedad, tanto a hombres como a mujeres se les han asignado responsabilidades diferentes. Por ejemplo, las mujeres siempre estuvieron relegadas a tareas del cuidado del hogar, maternidad y crianza de los hijos, mientras que las responsabilidades establecidas para los hombres eran netamente las económicas. La década de los ochenta trajo consigo muchos cambios económicos. A causa de las crisis latinoamericanas y al crecimiento del desempleo en la región, las mujeres tuvieron que incorporarse al mercado laboral, lo cual produjo un cambio en la estructura familiar y también en el rol que se les había dado hasta ese momento. La participación laboral de las mujeres en la región se estabilizó en la década de los noventa y con ello mejoraron sus condiciones laborales (onU Mujeres, 2017); sin embargo, hay evidencias de que en Ecuador existe discriminación salarial por género. Por ello, es importante analizar la situación actual de la mujer en el país de acuerdo a una perspectiva histórica.

El primer indicador a analizar es el de población económicamente activa ${ }^{1}$ (PEA), dentro del cual se excluyen a las personas que realizan labores domésticas, estudiantes, jubilados o personas que no pueden laborar a causa de algún tipo de discapacidad crónica. Lastimosamente, no se cuenta con un rubro específico de las personas que se dedican al quehacer doméstico, razón por la cual las cifras del mercado laboral ecuatoriano subestiman la participación laboral femenina. De acuerdo a datos de la Encuesta Nacional de Empleo, Desempleo y Subempleo (ENEMDU) (INEC, 2017), la PEA masculina para el año 2017, con respecto al total, se ubicó en $57,71 \%$, mientras que la PEA femenina estuvo en $42,29 \%$. Estos datos, respecto con la mujer, han mejorado desde el año 2010, pues en aquellas épocas la PEA masculina se encontraba en 60,57\%, mientras que la PEA femenina estuvo en $39,43 \%$. Se evidencia que las mujeres han tenido un mejoramiento, pero es evidente que frente al incremento marginal aún existe una brecha que cubrir.

Un segundo indicador importante es la categoría de empleo adecuado. ${ }^{2}$ La figura 1 muestra 
dicho indicador según el género en Ecuador, entre los años 2010 a 2017. De acuerdo a datos de la ENEMDU (INEC, 2017), esta tasa, para el caso masculino, fue de 67,60\%; mientras tanto, para el caso femenino se ubicó en $32,40 \%$. Si se comparan estos datos con los obtenidos en el año 2010 se puede observar que este indicador ha variado positivamente, aunque en un mínimo de $0,23 \%$ para el género femenino. Otro de los indicadores analizados - el tercero- es el subempleo, ${ }^{3}$ y que dentro de la figura 2 se muestra su evolución histórica entre los años 2010 a 2017. Con respecto al año 2017, la tasa de subempleo masculina se ubicó en 58,20\% en relación al total, y la tasa de subempleo femenina fue de $41,80 \%$. En comparación con los datos obtenidos en el año 2010, la tasa masculina fue de $61,85 \%$ y la femenina de $38,15 \%$; lo que evidencia una evolución para el caso masculino, no obstante un deterioro para el caso femenino. Un cuarto indicador es la tasa de desempleo, ${ }^{4}$ que, en la figura 3 , se muestra su evolución histórica para el periodo ya especificado. Sobre la evidencia de estos datos se observa que la tasa de desempleo de las mujeres ha sido mayor a la tasa de desempleo de los hombres dentro de estos siete años comparativos. De acuerdo a los datos de la ENEMDU, para el año 2017, la tasa masculina fue de 44,81\% mientras que la tasa femenina se ubicó en 55,19\%; para el caso femenino, lastimosamente, es la cifra más alta de desempleo alcanzada entre los años 2010 a 2017. Por consiguiente, luego de analizar el desarrollo negativo de este indicador, para el caso femenino se concluye que les hace muy complicado encontrar un empleo que alcance las expectativas no solo salariales, sino con respecto a la calidad de empleo al que acceden. Estos resultados denotan, también, que la brecha dentro del mercado laboral aún persiste entre mujeres y hombres.

Otro indicador analizado (el quinto) es el referente a grupos de ocupación por género en Ecuador, específicamente enfocado en el año 2017. En la tabla 1 se puede ver que la mayoría de profesiones están dominadas por el género masculino, aunque se resaltan tres ocupaciones ampliamente dominadas por el género femenino como son profesionales científicos, comerciantes y trabajadores no calificados. En el caso de científicos e intelectuales, las mujeres alcanzaron un $56,25 \%$ frente al masculino; para el caso de comerciantes y trabajadores en servicios alcanzaron el 59,60\%; y, la tercera ocupación, en el caso de trabajadores no calificados, registra un porcentaje igual a 51,43\%. Existen otras ocupaciones en donde la participación del género femenino es casi nula; así, por ejemplo, están operadores de maquinaria, cuya participación femenina fue de 5,16\% versus al 94,84\% de participación masculina; y dentro del campo de fuerzas armadas y ejército, la presencia de mujeres fue de 1,15\% frente al 98,85\% de participación masculina.

Dentro de este análisis conjunto, otro indicador considerado fue el nivel de ingreso salarial por género para el caso ecuatoriano. Como fue expuesto dentro del marco teórico, la discriminación salarial ocurre cuando una persona que pertenece a un grupo de autoidentificación percibe un salario diferente a causa de ello. Al analizar los datos, entre los años 2010 a 2017, se ha observado la existencia de discriminación salarial, jugando en contra del género femenino, y ha sido constante en términos porcentuales a lo largo del periodo citado. Para el año 2017, en promedio, los ingresos femeninos fueron menores en aproximadamente USD 104 con respecto a los salarios masculinos. En conclusión, es aquí en donde tienen que actuar las políticas públicas, impulsadas desde los organismos pertinentes para dar una solución a este mal. Las actuales condiciones femeninas no solo les afectan a ellas propiamente, sino también a las 
Tabla 1. Grupo de ocupación por género (2017)

\begin{tabular}{lcc}
\hline OCUPACIONES & HOMBRES & MUJERES \\
\hline Personal directo/Administración pública & $62,87 \%$ & $37,13 \%$ \\
\hline Profesionales científicos & $43,75 \%$ & $56,25 \%$ \\
\hline Técnicos de nivel medio & $55,02 \%$ & $44,98 \%$ \\
\hline Empleados de oficina & $50,07 \%$ & $49,93 \%$ \\
\hline Comerciales & $40,40 \%$ & \\
\hline Trabajadores calificados agropecuarios & $72,69 \%$ & \\
\hline Oficiales operarios y artesanos & $77,58 \%$ & $27,31 \%$ \\
\hline Operadores de maquinaria & $94,84 \%$ & \\
\hline Trabajadores no calificados & $58,57 \%$ & $5,16 \%$ \\
\hline Fuerzas armadas & $98,85 \%$ & \\
\hline
\end{tabular}

Fuente: ENEMDU (2010-2017), INEC (en línea). Elaboración: propia.

Tabla 2. Grupos de ocupación femenina por autoidentificación 2017

\begin{tabular}{|c|c|c|c|c|c|}
\hline OCUPACIONES & Indígena & Afroecuatoriano & Montubio & Mestizo & Blanco \\
\hline Personal directo/administración pública & $1,56 \%$ & $3,92 \%$ & $0,00 \%$ & $90,07 \%$ & $4,01 \%$ \\
\hline Profesionales científicos & $2,48 \%$ & $4,48 \%$ & $2,06 \%$ & $89,60 \%$ & $1,32 \%$ \\
\hline Técnicos de nivel medio & $2,08 \%$ & $2,77 \%$ & $1,38 \%$ & $92,11 \%$ & $1,67 \%$ \\
\hline Empleados de oficina & $1,39 \%$ & $3,01 \%$ & $0,60 \%$ & $92,77 \%$ & $1,83 \%$ \\
\hline Comerciantes & $4,75 \%$ & $4,84 \%$ & $3,35 \%$ & $85,12 \%$ & $1,80 \%$ \\
\hline Trabajad. calificados agropecuarios & $26,86 \%$ & $2,38 \%$ & $6,68 \%$ & $63,26 \%$ & $0,80 \%$ \\
\hline Oficiales operarios y artesanos & $2,41 \%$ & $1,63 \%$ & $2,95 \%$ & $92,03 \%$ & $0,87 \%$ \\
\hline Operadores de maquinaria & $7,49 \%$ & $3,91 \%$ & $0,26 \%$ & $86,21 \%$ & $2,12 \%$ \\
\hline Trabajadores no calificados & $20,59 \%$ & $3,86 \%$ & $4,27 \%$ & $69,52 \%$ & $1,57 \%$ \\
\hline Fuerzas armadas & $0,00 \%$ & $0,00 \%$ & $0,00 \%$ & $100,00 \%$ & $0,00 \%$ \\
\hline
\end{tabular}

Fuente: ENEMDU (2010-2017), (INEC en línea). Elaboración: propia.

familias quienes dependen de este ingreso para mantener un nivel de vida decente.

Cuando nos referimos a la existencia de discriminación salarial, no solo hacemos alusión a lo que sucede entre hombres y mujeres, sino también a lo que ocurre dentro del mismo género femenino según el grupo etario al que pertenecen. Para esto se analizaron dentro de este género los mismos indicadores que ya fueron anteriormente planteados, es decir, PEA, empleo adecuado, subempleo, desempleo, grupos de ocupación y nivel salarial. Después de este análisis se puede deducir que en todas las categorías las mujeres mestizas absorben las mejores condiciones. Por ejemplo, según datos del 2017 (INEC, 2017), el 80,09\% de la PEA femenina está conformada por mujeres mestizas; en la categoría de empleo adecuado se encuentran en un $88,84 \%$; con respecto al subempleo en un $79,85 \%$; mientras que en la categoría de desempleo con un $82,79 \%$. En la tabla 2 se muestran los diez grupos de ocupación, y en ellos los pesos por grupo etario o autoidentificación para el año 2017 dentro de Ecuador.

Como se puede observar en la tabla 2, todas las ocupaciones están dominadas por las mujeres que se autodenominan como mestizas, claro está, por la debida realidad étnica ecuatoriana. No obstante, llama la atención que en la mayor parte de estas ocupaciones la proporción de las mujeres mestizas está en alrededor del 90\% o superior a ésta; sin embargo, en la categoría de trabajadores agropecuarios y pesqueros su porcentaje es menor con el 63,26\%, seguido por las mujeres indígenas con un $26,86 \%$. Otra de las categorías en donde se ve reducida su jerarquía son las ocupaciones elementales, donde su nivel de participación fue de 69,52\% frente al 20,59\% 
de las mujeres indígenas. Un dato que resalta es sobre las fuerzas armadas; en el año 2017 el $100 \%$ de mujeres que se enlistaron en sus filas fueron mestizas, lo que hace necesario revisar las políticas de ingreso o bien conocer por qué las mujeres de otras etnias no lo han logrado.

En relación a la categoría de ingreso salarial, de acuerdo a la información arrojada por las ENEMDU, y a partir de un análisis de la evolución histórica entre los años 2010 y 2017 por autoidentificación femenina, las mujeres blancas poseen el mayor nivel de ingresos; para el año 2017 ganaron en promedio USD 562,79, es decir, USD 124,49 más que las mestizas (USD 438,30). A pesar de que los índices de empleo de las mujeres blancas son bajos en comparación con las mestizas, llama la atención que este grupo étnico cuenta con los más altos ingresos laborales. Por otro lado, las mujeres con los ingresos más bajos son las indígenas; de hecho, en el año 2010 el ingreso de este grupo fue de USD 160,97, mientras que para el 2017 fue de USD 256,94. Con estos resultados queda demostrado que dentro del grupo de mujeres también existen diferencias salariales a causa de la etnicidad de la que proceden.

\section{ANÁLISIS EMPÍRICO O CUANTITATIVO}

La discriminación salarial por razón de género y por autoidentificación dentro del campo femenino, se ha visibilizado en la sección anterior. Sin embargo, con el análisis empírico se pueden determinar valores más concisos. Por tal razón, dentro de esta sección se plantean dos modelos de análisis de varianza (ANOVA), desde donde se analizan la relación existente entre el ingreso salarial frente a variables como género (hombre, mujer) y, posteriormente, según grupos de autoidentificación para el caso femenino. Para este análisis, se utilizó la ENEMDU 2017. Cabe señalar que ésta es una encuesta por muestreo probabilístico, cuyo propósito principal es la medición y seguimiento del empleo, desempleo y la caracterización del mercado de trabajo, con lo cual da a conocer sobre las actividades económicas y fuentes de ingreso de la población en general (INEC, 2017).

Sobre la encuesta, en relación al año 2017, fue necesaria la identificación, depuración y recodificación y la creación de nuevas variables; posteriormente se utilizó el software estadístico Stata versión 14 para el tratamiento de datos. Con respecto al primer modelo, se eliminó la información incompleta y se generó una nueva variable dummy denominada $d \_m u j e r e s$ para observar el diferencial en los salarios entre hombres y mujeres. Sobre el segundo modelo, se trabajó solamente con los datos de las mujeres, aunque se crearon cuatro variables dummies correspondientes a mujeres indígenas, afroecuatorianas, montubias y blancas; en general, esto permite conocer el diferencial salarial entre mujeres según su autoidentificación. Debe mencionarse que se trabajó con un factor de expansión que sirve para expandir los datos de la encuesta de hogares hacia la población en estudio. Se destaca adicionalmente que la unidad económica básica fue el individuo y que los ingresos fueron trimestrales.

PRIMER MODELO: DISCRIMINACIÓN SALARIAL POR GÉNERO

El primer modelo plantea la discriminación salarial con respecto al género. La variable dependiente se corresponde al ingreso salarial tanto de hombres y mujeres, mientras que la variable independiente es dicotómica o dummy, y tomará el valor de la categoría analizada según 
Tabla 3. Resultados del primer modelo

\begin{tabular}{lc}
\hline INGRESO DEL TRABAJO & COEFICIENTE \\
\hline \multirow{2}{*}{ d_mujeres } & $-104,8753^{* * *}$ \\
\hline \multirow{2}{*}{ constante } & $-78,836$ \\
\hline Número de observaciones & $526,6828^{* * *}$ \\
\hline R-squared & $-52,336$ \\
\hline Fisher & 43,378 \\
\hline
\end{tabular}

Nota: El modelo fue corregido por heterocedasticidad, sin que exista presencia de multicolinealidad (apéndice A). Fuente:

ENEMDU (2017), INEC (en línea). Elaboración: propia.

su género (o: hombre, 1: mujer). Así entonces, luego de generados los valores para el primer modelo, ${ }^{5}$ queda planteada la ecuación [1]. De acuerdo a los resultados arrojados se tiene que los hombres tienen un ingreso de USD 526,68 en promedio, mientras que el de las mujeres es de USD 421,80 $(526,68-104,88=421,80)$.

Es decir, las mujeres ganan en promedio USD 104,88 menos que los hombres según los datos del año 2017 en Ecuador. Con estos resultados se demuestra la existencia de discriminación salarial por razón de género en el país dentro del periodo mencionado, y que en el mercado laboral ecuatoriano existe una desventaja para el género femenino.

$\mathrm{Y}=526,68-104,88 \mathrm{X}$

SEGUNDO MODELO: DISCRIMINACIÓN SALARIAL FEMENINA POR AUTOIDENTIFICACIÓN

El segundo modelo plantea ahora la discriminación salarial, dentro del segmento femenino, pero haciendo énfasis en la autoidentificación. La variable dependiente se corresponde con el ingreso salarial femenino. A la vez, las variables independientes se corresponde con dummies, las mismas que toman el valor de la categoría analizada de: 1-indígena, 2-afrodescendiente, 3-montubio, 5-blanco; en que el análisis se hará con respecto al grupo mayoritario de mestizo, por lo que se obvia dentro de estas especificaciones a dicho grupo. De acuerdo a los resultados obtenidos se plantea la ecuación [2]. Según estos cálculos, ${ }^{6}$ se obtiene como resultado que las mujeres mestizas en promedio tienen un ingreso laboral de USD 438,41; mientras tanto, las mujeres indígenas en promedio obtuvieron un salario de USD 256,95 $(438,41-181,46=256,95)$, con lo cual sufren una diferencia de USD 181,46 con respecto a las mestizas.

Con respecto a las mujeres afroecuatorianas, tienen en promedio un ingreso laboral de USD 408,91 $(438,41-29,50=408,91)$, es decir, sufren una diferencia de USD 29,50 con respecto a las mestizas. Por otro lado, las mujeres montubias tienen en promedio un ingreso laboral de USD 276,52 $(438,41-161,89=276,52)$, es decir, sufren una diferencia de USD 161,89 con respecto a las mestizas. Por último, las mujeres blancas en promedio tienen un ingreso laboral de USD $562,79(438,41+124,38=562,79)$, con lo cual poseen una diferencia positiva de USD 124,38 con respecto a las mestizas.

Bajo este análisis, se demuestra que también existe también discriminación salarial femenina por autoidentificación en Ecuador, según los datos del año 2017. Cabe resaltar que en 
el país ya se ha tratado este tema de igualdad de oportunidades, y las reformas legales dan prueba de ello, no obstante se evidencia a través de los modelos planteados que aún queda camino por recorrer.

$$
Y=438,41-182,46 X_{1}-29,50 X_{2}-161,89 X_{3}+124,39 X_{5}
$$

\section{DESAFÍOS Y POLÍTICAS SECTORIALES}

Históricamente, el mercado laboral ecuatoriano ha estado asociado con altas tasas de desempleo y subempleo, baja calidad del empleo, relaciones laborales precarias y desconocimiento de los derechos laborales por parte de los trabajadores. Otra de las características de este mercado es que gran parte de la PEA se encuentra inmersa en el sector informal. Por otro lado, la normativa que nos rige ha variado según la orientación ideológica del gobierno de turno y de la coyuntura económica en la que el país se encuentre. Así, desde el regreso a la democracia en 1979, el Ecuador ha tenido normas laborales protectoras y procesos de flexibilización laboral. Con la puesta en escena de la última Constitución de 2008, se han emprendido una serie de reformas en materia laboral con las que se pretende eliminar las diferencias existentes, y en las que se incluyen nuevas categorías como el trabajo de cuidado y el autoempleo; a la vez se busca promover la obligatoriedad de la afiliación a la seguridad social.

En este sentido, en la Constitución (2008), en su artículo 11, literal 2, se hace mención a que ningún ciudadano puede ser discriminado, y se menciona también que cualquier forma de discriminación será sancionada por la ley. En el artículo 65 se hace mención a que el Estado promoverá la representación equitativa de hombres y mujeres dentro de empleos en la función pública y dentro de la política también. En el artículo 70, se hace referencia a que el Estado formulará y ejecutará políticas públicas con enfoque de género para alcanzar la igualdad entre hombres y mujeres. En relación al Régimen de Desarrollo, en el artículo 325, se extiende la protección laboral al trabajo autónomo, de autosustento y de cuidado humano. En los artículos 329, 330, 331 y 332 se garantiza la no discriminación y las medidas de acción afirmativa para ciertos grupos que históricamente han sido víctimas de discriminación, tales como jóvenes, mujeres, personas pertenecientes a las distintas comunidades, pueblos y nacionalidades del Ecuador.

En el Código de Trabajo, por otro lado, en el Capítulo vi De los salarios, de los sueldos, de las utilidades y de las bonificaciones y remuneraciones adicionales, en el parágrafo $1 .^{\circ}$ De las remuneraciones y sus garantías, en su artículo 79, se establece la igualdad en las remuneraciones; desde luego, su establecimiento no contempla algún tipo de discriminación ya sea por edad, género, etnia, origen social, idioma, religión, posición económica, orientación sexual o bajo otras formas de discriminación. Conjuntamente, en los dos últimos planes de desarrollo nacionales se garantiza además el acceso al trabajo estable, justo y digno en todas sus formas, además privilegia el trabajo sobre el capital, se garantizaba la igualdad salarial entre hombres y mujeres, y en conjunto se asegura el propio sustento y autonomía de cada ciudadano. Asimismo, con los planes se pretende abolir la división sexual del trabajo y garantizar a todas las personas el acceso equitativo a la educación y al empleo.

Con estos antecedentes se observan los esfuerzos realizados al interior del país por cambiar 
Tabla 4. Resultados del segundo modelo

\begin{tabular}{lc}
\hline INGRESO DEL TRABAJO & COEFICIENTE \\
\hline \multirow{2}{*}{ d_mujeres } & $-104,8753^{* * *}$ \\
\hline \multirow{2}{*}{ constante } & $-78,836$ \\
\hline Número de observaciones & $526,6828^{* * *}$ \\
\hline R-squared & $-52,336$ \\
\hline Fisher & 43,378 \\
\hline
\end{tabular}

Nota: El modelo fue corregido por heterocedasticidad, sin que exista presencia de multicolinealidad (apéndice B). Fuente:

ENEMDU (2017), INEC (en línea). Elaboración: propia.

Tabla 5. Resultados del primer modelo corregido heterocedasticidad con la utilización de métodos robustos

\begin{tabular}{lc}
\hline INGRESO DEL TRABAJO & COEFICIENTE \\
\hline \multirow{2}{*}{ d_mujeres } & $-104,8753^{* * *}$ \\
\hline \multirow{2}{*}{ constante } & $-78,836$ \\
\hline Número de observaciones & $526,6828^{* * *}$ \\
\hline R-squared & $-52,336$ \\
\hline Fisher & 43,378 \\
\hline
\end{tabular}

Elaboración: autores.

la situación señalada y que se ha evidenciado en las dos secciones anteriores. No obstante, para Ribas (2000) hace falta la transversalización del término género bajo una perspectiva de equidad, no solo en el ámbito económico, sino también en el laboral y social. Cabe indicar que la equidad de género es un componente esencial del proceso de desarrollo económico, de integración social y de consolidación de sociedades más democráticas. Así entonces, la construcción de la dimensión de género es importante para poder erradicar conductas discriminatorias en el ámbito laboral y profesional, lo que ayudará también a que la sociedad cambie la percepción de los roles que se les ha dado tanto a hombres como mujeres. De esta manera, se espera que con el pasar del tiempo se consiga la igualdad y equidad de oportunidades, eliminar la segregación ocupacional que experimentan las mujeres, incrementar los niveles de productividad y competitividad, y con ello lograr un crecimiento sostenido (Maurizio, 2010).

La Organización Internacional del Trabajo (огт) incorporó la perspectiva de género en el año de 1995 durante la Cuarta Conferencia Mundial sobre la Mujer en Beijing. En el año de 1999, creó, además, su propia política con respecto a la igualdad entre hombres y mujeres, y se destacó en tres frentes como son la dotación de personal, la sustancia y la estructura. Lastimosamente, la incorporación de la perspectiva de género no ha sido bien entendida y trabajada en la región, especialmente en Ecuador, lo que ha generado que no se alcancen los resultados esperados. La oit en su informe Guía de recursos sobre las cuestiones de género en las políticas de empleo y del mercado de trabajo. Empoderamiento económico de la mujer e igualdad de género, brinda ciertas recomendaciones para que la introducción de dicho tema sea más efectiva. La primera se fundamenta en la adopción de un enfoque más estratégico; la 
segunda, en el fortalecimiento del apoyo institucional; la tercera, en la atención a las políticas internas de recursos humanos; y, la cuarta, sobre la puesta a disposición de recursos para apoyar el análisis.

En términos generales, la ort considera importante la transversalidad de la igualdad de género y la justifica mediante dos argumentos. El primero de ellos, la equidad basada en los derechos; es decir, la discriminación que sufren las mujeres dentro del entorno laboral tiene que ser considerada y tratada como una cuestión de derechos humanos y justicia social. El segundo argumento, la eficiencia económica; en que las mujeres pueden llegar a desempeñar un papel decisivo como agentes de cambio en la sociedad. La igualdad no solo es considerada como un derecho que tienen las personas dentro de la sociedad para cambiar su realidad, sino también es una herramienta necesaria para lograr crecimiento económico, reducción de la pobreza y para la contribución del progreso del país.

\section{REFLEXIONES FINALES}

Dentro de este trabajo se analizó y se llegó a medir en Ecuador, por un lado, la discriminación salarial por razón de género y, por otro, la discriminación salarial dentro del segmento femenino según su autoidentificación; así también se estudió entre los años 2010 a 2017 la evolución histórica de los diferentes indicadores que demuestran esta realidad ecuatoriana. Para ello, se partió de los datos proporcionados por la Encuesta Nacional de Empleo, Desempleo y Subempleo (ENEMDU). Con el fin de medir la discriminación salarial según los casos indicados se plantearon dos modelos matemáticos. El primero permitió analizar la discriminación salarial por razón de género, mientras que el segundo permitió analizar la discriminación salarial del grupo femenino por su autoidentificación. Según estos resultados, se puede afirmar que en Ecuador sí existe discriminación salarial con respecto a la mujer, y dentro de este género dicha discriminación se acrecienta según su autoidentificación.

Además, con respecto al análisis de los datos obtenidos se ha evidenciado la existencia de dos fuerzas laborales (masculina y femenina); ambas compiten entre sí en el mercado laboral. Sin embargo, en el desarrollo de sus actividades la fuerza laboral femenina es más vulnerable a sufrir discriminación salarial o económica, solamente por su condición de mujer. De hecho, en relación a las conclusiones obtenidas a partir del primer modelo, se evidencia que el salario de los hombres en promedio, durante el año 2017 fue de USD 526,68, mientras que el de las mujeres fue de USD 421,8; es decir, existió una diferencia salarial de USD 104,88 a favor de los hombres. Para el segundo modelo, con el establecimiento de cuatro variables dummies, las que representaron los grupos de autoidentificación femenino (afroecuatoriano, indígena, montubio y blanco), se concluye además la existencia la discriminación salarial femenina por autoidentificación.

Sobre los resultados arrojados de este segundo modelo se observó que el salario de las mujeres mestizas, en promedio para el año 2017 , fue de USD 438,30. A la vez, las mujeres indígenas tuvieron, en promedio, un ingreso laboral de USD 256,94; es decir, contemplaron una diferencia de USD 181,36 menos que el grupo de mestizas. Acerca de las mujeres afroecuatorianas, en promedio, tuvieron un ingreso laboral de USD 408,91, con lo cual sufren una diferencia de 
Tabla 6. Resultados del segundo modelo corregido heterocedasticidad con la utilización de métodos robustos

\begin{tabular}{|c|c|c|c|c|c|c|}
\hline $\begin{array}{l}\text { INGRESO DE } \\
\text { TRABAJO }\end{array}$ & COEFICIENTE & $\begin{array}{l}\text { ERROR } \\
\text { ESTÁNDAR }\end{array}$ & $\mathrm{T}$ & $\mathrm{P}>1 \mathrm{t} 1$ & \multicolumn{2}{|c|}{$\begin{array}{l}\text { 95\% CONFIANZA } \\
\text { DEL INTERVALO }\end{array}$} \\
\hline d_mujeres & -1.048 .753 & 78.836 & -13.30 & 0.000 & -1.203 .273 & -894.234 \\
\hline _constante & 5.266 .828 & 52.336 & 100.64 & 0.000 & 5.164 .248 & 5.369 .407 \\
\hline $\begin{array}{l}\text { Número de } \\
\text { observaciones }\end{array}$ & 43,378 & & & & & \\
\hline R-squared & 0.0074 & & & & & \\
\hline Fisher & 176.97 & & & & & \\
\hline
\end{tabular}

Elaboración: autores.

USD 29,39 con respecto a las mestizas. Sobre las mujeres montubias, ellas tuvieron en promedio un ingreso laboral de USD 276,51; es decir, una diferencia negativa de USD 161,79 con respecto a las mestizas. Por último, las mujeres blancas, en promedio, tuvieron un ingreso laboral de USD 562,79; esto es, obtuvieron un salario de USD 124,49 superior con respecto a las mujeres autodenominadas mestizas.

Frente a esta realidad ecuatoriana de discriminación, en el año 2008, con la creación de una nueva Constitución, el Estado consagra nuevas normas con respecto a los derechos y principios de los trabajadores, protección social, y se reconoce además al trabajo como eje importante de la economía ecuatoriana. Desde luego, son muchos los artículos donde se garantiza la no discriminación de todo tipo, así como el acceso equitativo a puestos de trabajo, la ejecución de políticas públicas con enfoque de género, la equidad en los salarios y, entre otros, la afiliación a la seguridad social. Conjuntamente, el Código del Trabajo garantiza también estos derechos dentro de la legislación ecuatoriana, y lo hace a través del planteamiento de remuneraciones sin ningún tipo de discriminación. Por su parte, en los planes de desarrollo nacional se establecieron ciertas normas como, por ejemplo, el acceso al trabajo estable en todas sus formas y se apunta a la abolición de la división sexual del trabajo y reconocer el trabajo no remunerado.

Así, dentro del país se han creado varios mecanismos, sean leyes o normas, que defienden la igualdad de oportunidades entre hombres y mujeres, pero el camino por recorrer es bastante largo, y queda seguir con este monitoreo permanente para ir corrigiendo o estableciendo nuevas normativas legales. Para finalizar, se espera que este trabajo se convierta en un medio de consulta y que sea una radiografía sobre la realidad por la cual transita el género femenino dentro del mundo laboral; a la vez, queda también reflexionar o preguntarnos acerca del impacto negativo que conlleva la discriminación salarial dentro del desarrollo humano y social.

\section{NOTAS}

1 En la PEA se considera « [...] [A] todas las personas de 15 años en adelante que al menos trabajaron una hora durante la semana en la cual se hizo la medición, o que en algún momento de su vida trabajaron, o que actualmente se encuentren en paro, pero estén disponibles para reincorporarse al mercado laboral» (INEC, 2017).

2 El empleo adecuado involucra a todas las personas de 15 años en adelante que se dedicaron a la realización de alguna actividad laboral durante la semana de referencia, además, se refiere también a todas las personas que perciben salarios iguales o superiores al salario mínimo y que trabajan alrededor de 40 
horas o más (INEC, 2017).

3 Se define como subempleo a todas las personas que durante la semana de referencia percibieron salarios menores al salario mínimo y que, adicional a ello, no cumplieron con las 40 horas que están establecidas en la jornada laboral (ibíd.).

4 En relación al desempleo « [...] [S]e refiere a las personas de 15 años en adelante que durante el período de referencia no estuvieron empleados y que no han tenido empleo durante un periodo establecido de tiempo pero se encuentran disponibles para hacerlo» (INEC, 2017).

5 Luego de corrido el primer modelo se obtuvo la tabla adjunta, la misma que se decidió ponerla al final con el fin de dotar a la lectura de una mayor fluidez (ver Tabla 3). Y véanse los resultados del primer modelo corregido heterocedasticidad con la utilización de métodos robustos (ver Tabla 5).

6 La tabla de resultados del segundo modelo queda a continuación adjunta, y que, de igual manera, se decidió ponerla al final con el fin de dotar a la lectura de una mayor fluidez (ver Tabla 4). Y véanse los resultados del segundo modelo corregido heterocedasticidad con la utilización de métodos robustos (ver Tabla 6).

\section{REFERENCIAS}

Aigner, D. and Cain, G. (January, 1977). Statistical theories of discrimination in labor markets. Industrial and Labor Relations Review, 3o(2), 175-187. Recuperado de http://econ2.econ.iastate.edu/classes/econ321/rosburg/Aigner\%2oand\%20Cain\%20-\%2oStatistical\%2oTheories\%20of\%20 Discrimination\%2oin\%2oLabor\%2oMarkets.pdf

Asamblea Nacional Constituyente. (2008). Constitución del Ecuador. Manabí, Ecuador: Asamblea Nacional Constituyente.

Becker, G. (1971). The economics of discrimination. Chicago, UsA: The University of Chicago.

Eurostat Static Explained. (April, 2017). Eurostat. Recuperado de http://ec.europa.eu/eurostat/statisticsexplained/index.php/Wages_and_labour_costs/es\#M.C3.A1s_informaci.C3.B3n_de_Eurostat

Gross, E. (Autumn, 1968). Plus CA Change...? The sexual structure of occupations over time. Social problems, 16(2), 198-208. Recuperado de https://academic.oup.com/socpro/article-abstract/16/2/19 $8 / 1683956$ ? redirectedFrom $=$ fulltext

Hopenhayn, M. y Bello, A. (2000). Tendencias generales, prioridades y obstáculos en la lucha contra el racismo, la discriminación racial, la xenofobia y las formas conexas de intolerancia. América Latina y el Caribe. Cepal. Recuperado de https://www.cepal.org/prensa/noticias/comunicados/4/5534/xeno.pdf.

Instituto Ecuatoriano de Estadísticas y Censos (INEC). (2017). Reporte de Economía Laboral. Ecuador en cifras. Recuperado de http://www.ecuadorencifras.gob.ec/documentos/web-inec/EMPLEO/2017/ Diciembre/122017_M.Laboral.pdf

Instituto Ecuatoriano de Estadísticas y Censos (INEC). (2017, mayo 24). Instituto Ecuatoriano de Estadísticas y Censos. Recuperado de http://www.ecuadorencifras.gob.ec/censo-de-poblacion-y-vivienda/

Instituto Ecuatoriano de Estadísticas y Censos (INEC). (2017, mayo). Encuesta Nacional de Empleo, Desempleo y Subempleo-enemdu. Ecuador en cifras. Recuperado de http://www.ecuadorencifras.gob.ec/documentos/webinec/EMPLEO/2017/Diciembre/122017_M.Laboral.pdf

Itzkowich, C. y Torrecillas, V. (2017). El progreso de las mujeres en América Latina y el Caribe 2017. ONU Mujeres. Recuperado de https://www2.unwomen.org/-/media/field\%2ooffice\%2oamericas/documentos/publicaciones/2017/o7/un16017_web.pdf?la=es\&vs=4654

Keegan, J. (1994). A History of Warfare. En J. Keegan, A History of Warfare (pp. 166-167). New York, UsA: 
Random House.

Maurizio, R. (2010, junio). Enfoque de género en las instituciones laborales y las políticas del mercado de trabajo en América Latina. Cepal. Recuperado de https://repositorio.cepal.org/bitstream/ handle/11362/5333/S10o0313_es.pdf?sequence=1\&isAllowed $=y$

Naciones Unidas. (2001). Declaración Mundial contra el Racismo, la Discriminación Racial, la Xenofobia y las Formas Conexas, de Intolerancia. Recuperado de http://www.un.org/es/events/pastevents/ cmcr/durban_sp.pdf

Peñas, I. L. (1998). Discriminación salarial por razones de género: un análisis empírico del sector privado de España. Reis, (s d.), 171-196.

Ribas Bonet, M. A. (200o). Desigualdades de género en el mercado laboral: un problema actual. Recuperado de http://dea.uib.cat/digitalAssets/128/128260_4.pdf 
